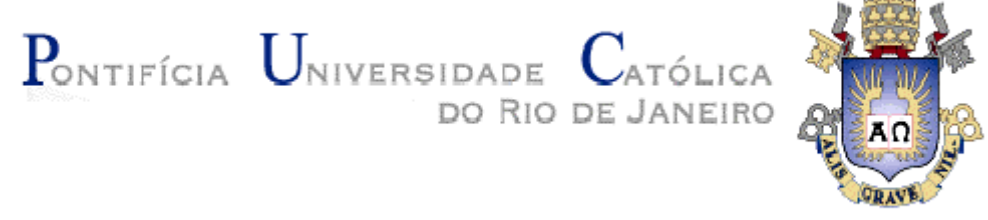

Diana da Veiga Mandelert

\title{
REPETÊNCIA EM ESCOLAS DE PRESTÍGIO: \\ Quanto, quando e como acontecem
}

Tese de Doutorado

Tese apresentada ao Programa de PósGraduação em Educação do Departamento de Educação da PUC-Rio como parte dos requisitos parciais para obtenção do título de Doutor em Educação.

Orientador: Prof ${ }^{a}$ Zaia Brandão

Rio de Janeiro

Junho de 2010 
Diana da Veiga Mandelert

\author{
REPETÊNCIA EM ESCOLAS DE PRESTÍGIO: \\ Quanto, quando e como acontecem
}

Tese apresentada como requisito parcial para obtenção do título de Doutor pelo Programa de Pós-Graduação em Educação do Departamento de Educação do Centro de Teologia e Ciências Humanas da PUC-Rio. Aprovada pela Comissão Examinadora abaixo assinada.

Prof $^{a}$ Zaia Brandão Orientadora

Departamento de Educação - PUC-Rio

Prof ${ }^{a}$ Isabel Alice Oswald Monteiro Lelis

Departamento de Educação - PUC-Rio

Prof ${ }^{a}$. Fatima Cristina de Mendonça Alves Departamento de Educação - PUC-Rio

Prof. Marcio da Costa

UFRJ

Prof $^{a}$. Maria Ligia de Oliveira Barbosa

UFRJ

Prof. Paulo Fernando C. de Andrade Coordenador Setorial do Centro de

Teologia e Ciências Humanas

Rio de Janeiro, 21 de junho de 2010. 
Todos os direitos reservados. É proibida a reprodução total ou parcial do trabalho sem autorização da universidade, da autora e do orientador.

\section{Diana da Veiga Mandelert}

Graduou-se em Direito pela UERJ em 1992 e em Pedagogia pela PUC-Rio em 2002. Mestre em Educação pela PUC-Rio em 2005. Atualmente é pesquisadora do SOCED - Grupo de Pesquisa em Sociologia da Educação da PUC-Rio, coordenado pela professora Zaia Brandão.

Ficha Catalográfica

Mandelert, Diana da Veiga

Repetência em escolas de prestígio: Quanto, quando e como acontecem / Diana da Veiga Mandelert ; orientadora: Zaia Brandão. - 2010.

158 f. : il. ; $30 \mathrm{~cm}$

Tese (doutorado)-Pontifícia Universidade Católica do Rio de Janeiro, Departamento de Educação, 2010.

Inclui bibliografia

1. Educação - Teses. 2. Pedagogia da repetência. 3. Reprovação. 4. Camadas médias e altas. 5. Escolas de prestígio. 6. Capital cultural. I. Brandão, Zaia. II. Pontifícia Universidade Católica do Rio de Janeiro. Departamento de Educação. III. Título. 
Para minhas queridas: Gaby e Lilian. 


\section{Agradecimentos}

À Zaia Brandão, professora e amiga: sem o seu apoio eu de fato não teria chegado ao fim.

Às escolas estudadas com seus excelentes profissionais, pela confiança em terem aberto a instituição para mim.

Aos meus filhos Sofia e Théo, amores incondicionais.

A José Maurício Arruti pela leitura amorosa e cuidadosa, além de ter aceitado discutir em todos os momentos de angústias e dúvidas.

Aos meus avós Fabio e Lygia queridos para sempre.

Aos meus pais, Anna Maria e Jean Pierre por terem sido leitores fiéis.

A todos os professores da Pós-Graduação em Educação da PUC-Rio, especialmente Fátima Alves por ter participado do projeto desde o seu nascedouro com críticas e sugestões fundamentais e Isabel Lelis

A todos os meus colegas da Pós-Graduação em Educação e do SOCED da PUCRio, em particular Jorge Cássio, Cristina Galvão, Glauco Aguiar e Hustana Vargas.

À Marylink Kupferberg, Estevão Mandelert e Marcelo Versiani colaboradores fundamentais pela manutenção da minha sanidade mental.

À Rosa Xavier Rodrigues, Débora Chagas e Joacir da Silva pelo apoio inestimável e funcionários do departamento de Educação sempre gentis e solícitos: Geneci, Janaína, Sandra, Nancy e Eduardo.

À minha família Buziana: André Valverde, Rogério Fulgêncio, Mag Paletta, Luciana Ramos, Andrea Weinberg, Mario Salonikius, Vinícius, Mabel, Mila, Erik, Bruno e Rafael.

Aos meus queridos de sempre: Clô e Flavio Franklin, Bernardo Mandelert e Valéria Celano, Fabiana Graça, Bel Osborne e Monica Urman.

Ao CNPq, FAPERJ e à PUC-Rio, pela ajuda financeira recebida. 


\section{Resumo}

Mandelert, Diana da Veiga; Brandão, Zaia. Repetência em escolas de prestígio: Quanto, quando e como acontecem. Rio de Janeiro, 2010. 158p. Tese de Doutorado - Departamento de Educação, Pontifícia Universidade Católica do Rio de Janeiro.

Esta pesquisa estudou o fenômeno da repetência em um contexto pouco pesquisado- colégios de prestígio e camadas médias e altas. Apoiada em Revel (1998), assumi como estratégia para o estudo o “jogo de escalas”. O trabalho se divide em quatro partes que, em escalas diferentes, buscam analisar o fenômeno: na sua magnitude e no processo concreto de reprovação. A primeira parte com os dados do PISA de 2006 realizei uma comparação do risco de atraso escolar no Brasil com outros três países: o México, a Argentina e a Colômbia, considerando o nível socioeconômico e a dependência administrativa da escola, controlando os resultados pelo desempenho dos estudantes em leitura. Vi que o risco de defasagem é muito maior no Brasil do que nos outros países mesmo entre os alunos das camadas mais altas. Na segunda parte construí um panorama do fluxo escolar em 15 escolas entre as de maior pontuação pelo ranqueamento feito pelo Enem. Constatei que todas as escolas reprovam seus alunos, mas que existem diferentes padrões de reprovação. $\mathrm{Na}$ terceira parte do trabalho analisei a entrada e a saída dos alunos ao longo dos 11 anos necessários para a formatura de uma geração em duas escolas de prestígio, fiz o que chamei de ciclo de série. Observei que os níveis de reprovação têm dois pontos altos: na $6^{\mathrm{a}}$ e na $7^{\mathrm{a}}$ série, e no $1^{\mathrm{o}}$ ano do ensino médio, praticamente inexiste a reprovação no $1^{\mathrm{o}}$ segmento do ensino fundamental. Para cada dez alunos que entram nessas escolas apenas três se formam sem reprovações. A última parte do trabalho foi a observação dos conselhos de classe de uma das escolas pesquisadas anteriormente. A enturmação das turmas e a manutenção das médias em zona de corte favoreceram a possibilidade de reprovar os alunos. No momento da avaliação final destaca-se a importância de um tipo específico de envolvimento familiar e a melhor adequação dos alunos das camadas médias no projeto de escolas de prestígio.

\section{Palavras-chave:}

Pedagogia da repetência; reprovação; camadas médias e altas; escolas de prestígio; capital cultural. 


\section{Abstract}

Mandelert, Diana da Veiga; Brandão, Zaia. (Advisor) Repetition in prestige's schools: amount, when and how they happen? Rio de Janeiro, 2010. 158p. Thesis - Departamento de Educação, Pontifícia Universidade Católica do Rio de Janeiro.

This dissertation analyzed the phenomenon of school failure in the light of an under-studied context: that of prestigious schools and middle to upper and classes. Based upon Revel (1998), we adopted the "game of scales" as our analytical strategy. The dissertation is divided into four parts which, in different measures, endeavor to study this phenomenon both in its magnitude and in the concrete process of school failure, by means of a microanalysis. Beginning with the PISA 2006 data, we compared the risk of being held back on probation in school in Brazil with that of three other countries: Mexico, Argentina and Colombia, considering their socioeconomic status and the schools' management dependencies, using the students' reading performance as a control measure. We found that the risk of probation holdup is much higher in Brazil than in other countries, even among students from high income social classes. In the second part of this study we charted the school attendance flux in 15 schools amongst those of highest standing in the Enem ranking. We found that all schools fail their students, but that there are different failure standards. In the third part of this study we sought to understand the entry and exit of students throughout the 11 years required for one generation to graduate by examining two prestigious schools, and establishing what we named a 'Cycle series'. We observed that school failure levels reach their three critical moments in the 6th and 7th grades, and that for the junior $\left(1^{\text {st }}\right)$ year of high school, they are practically nonexistent in the first segment of basic education. For every ten students entering these schools, only three graduate without ever failing a grade. The last part of this study was the observation of student evaluation board meetings in the previously surveyed schools. Class composition and maintenance in the cutoff section favored the possibility of failing students. Our final evaluation highlights the importance of a specific sort of family involvement and how middleincome students tend to fit in better with these prestigious schools’ projects.

\section{Key-words:}

Repetition; school failure; upper and middle classes; prestigious schools; cultural capital. 


\section{Sumário}

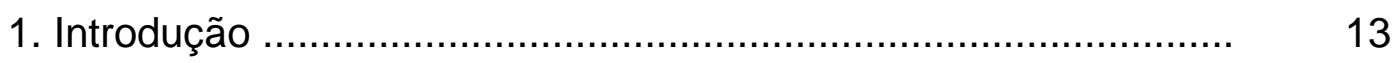

1.1. Desenhando o problema .................................................... 13

1.2. Estudos sobre os efeitos da repetência ............................... 19

1.3. Estudos no Brasil sobre o modelo alternativo à reprovação: as escolas em ciclos ............................................................ 24

1.4. Estudos sobre a repetência nas camadas médias e altas e em escolas de prestígio .......................................................... 26

1.5. Perguntas e métodos ........................................................ 32

2. A Defasagem escolar em camadas altas no Pisa 2006: Brasil, Colômbia, México e Argentina ..................................................... 36

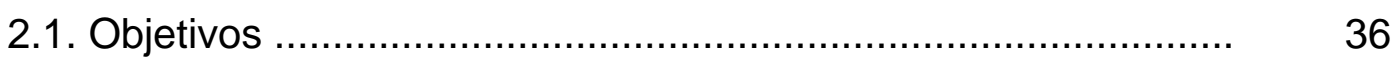

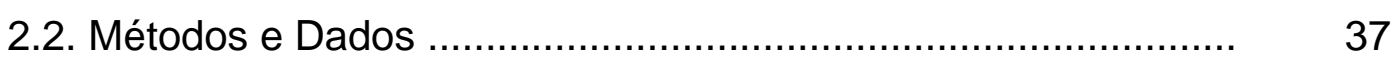

2.3. Análise exploratória ……….............................................. 40

2.4. Modelo de risco para a repetência ........................................... 41

3. Panorama da repetência em escolas de prestígio do Rio de Janeiro ........................................................................ 46

3.1. Objetivos e o Censo Escolar ............................................... 46

3.2. Observações Gerais .........................................................

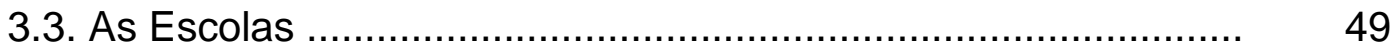

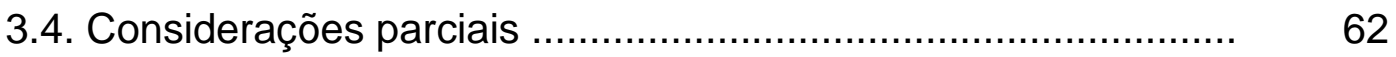

4. Escolas de prestígio: da qualidade à distinção ............................. 63

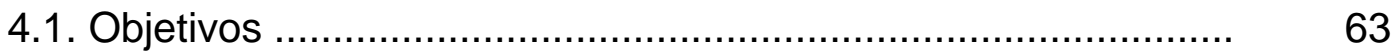

4.2. Dados e Tratamento ………………………….......................

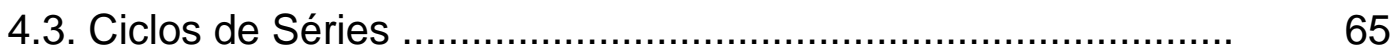

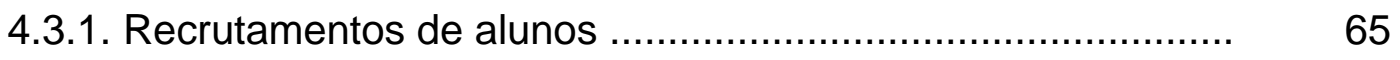

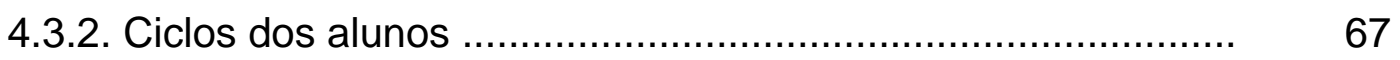

4.4. Coorte de turma ............................................................

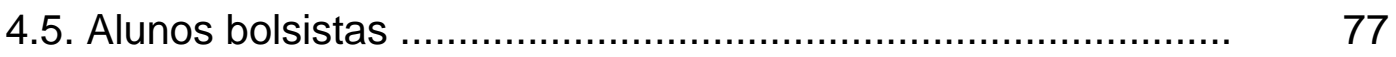

4.6. Considerações parciais ....................................................... 
5. Apreciações e Mecanismo de Corte: conselhos consultivos ....... 80

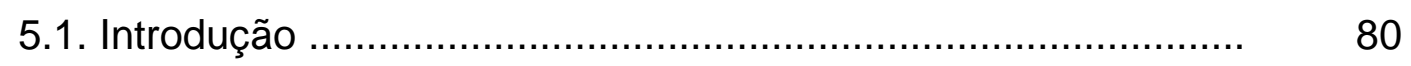

5.2. Questões sobre a observação dos conselhos de classe .......... 81

5.3. Conselhos Consultivos e Conselhos Deliberativos ................... 84

5.3.1. Descrição geral das reuniões ................................................ 86

5.3.2. Diferenças entre os conselhos do $8^{\circ} \mathrm{EF}$ e do $1^{\circ} \mathrm{EM}$............. 87

5.4. Conselhos Consultivos ............................................................ 89

5.4.1. "Perfis das turmas" ............................................................ $\quad 89$

5.4.2. Perfis dos alunos ............................................................ 93

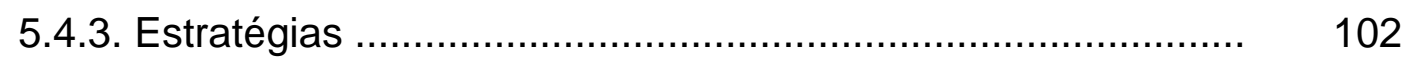

5.4.4. Zona de desconforto ...................................................... 107

6. Aprovações e Reprovações: conselhos deliberativos .................. 112

6.1. Descrição geral ................................................................ 112

6.2. Uma escola menos "excludente" ............................................ 113

6.3. Matérias que reprovam e que não reprovam .......................... 115

6.3.1. Ensino Fundamental .......................................................... 116

6.3.2. Ensino Médio .............................................................. 118

6.4. Conselhos pós-provas finais ............................................... 121

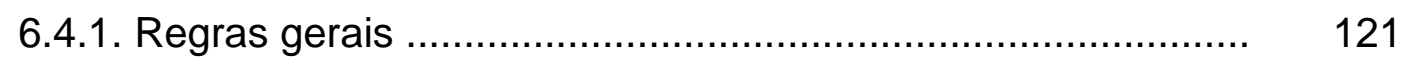

6.4.2. Os resultados e as regras pontuais ................................... 123

6.4.2.1. Ensino Fundamental ........................................................ 123

6.4.2.2. Ensino Médio ........................................................... 127

6.5. Conselhos pós-recuperação .................................................. 133

6.5.1. Ensino Fundamental ........................................................ 133

6.5.2. Ensino Médio ............................................................... 134

6.6. Reprovações Benéficas ....................................................... 136

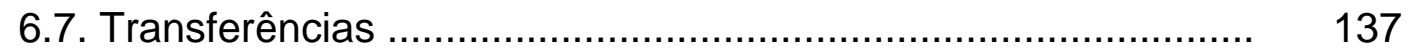

6.8. Considerações Parciais ....................................................... 138

7. Considerações Finais ........................................................... 142

8. Referências Bibliográficas ………………………................ 148

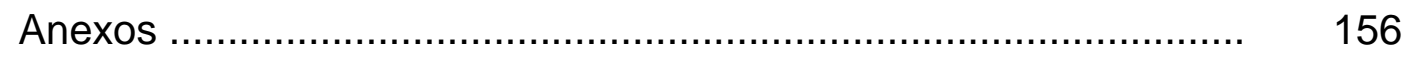




\section{Lista de Quadros, Tabelas e Gráficos}

Quadro 1 - Distribuição das tarefas de leitura, considerando os processos de compreensão textual

Tabela 1 - Valores médios de desempenho em leitura segundo os cinco aspectos avaliados

Tabela 2 - Valores médios das características dos alunos

Tabela 3 - Valores médios das características dos alunos defasados e não defasados

Tabela 4 - Parâmetros estimados pelos modelos de risco de estar defasado

Tabela 5 - Dados da escola A1 comparados com os dados do Censo Escolar de 2003 a 2005

Tabela 6 - Dados da escola A2 comparados com os dados do Censo Escolar de 2003 a 2005

Tabela 7 - Taxa de repetência dos quatro ciclos da escola A1 ....

Tabela 8 - Taxa de repetência dos quatro ciclos da escola A2

Tabela 9 - Frequência absoluta e relativa dos alunos da $1^{\text {a }}$ série e dos concluintes em cada coorte da escola A1

Tabela 10 - Frequência absoluta e relativa da zona de moradia dos alunos da coorte de 1997 da escola A1 conforme moradia na $1^{\mathrm{a}}$ série e os concluintes

Tabela 11 - Número de alunos que não passaram por nota em cada disciplina do $8^{\circ}$ EF nos anos de 2008 e 2009

Tabela 12 - Número de alunos que não passaram por nota em cada disciplina do $1^{\circ}$ EM nos anos de 2008 e 2009

Tabela 13 - Resultados pós provas finais do $8^{\circ}$ ano do ensino fundamental de 2008 - diferença entre a nota alcançada e a nota necessária 
Tabela 14 - Resultados pós provas finais do $8^{\circ}$ ano do ensino fundamental de 2009 - diferença entre a nota alcançada e a nota necessária

Tabela 15 - Resultados pós- provas finais do $1^{\circ}$ ano do ensino médio de 2008 - diferença entre a nota alcançada e a nota necessária

Tabela 16 - Resultados pós- provas finais do $1^{\circ}$ ano do ensino médio de 2009 - diferença entre a nota alcançada e a nota necessária

Tabela 17 - Resultados pós-recuperação do $1^{0}$ ano do ensino médio de 2008 - diferença entre a nota alcançada e a nota necessária

Tabela 18 - Resultados pós-recuperação do $1^{\circ}$ ano do ensino médio de 2009 - diferença entre a nota alcançada e a nota necessária

Tabela 19 - Motivos dos pedidos de transferência do $8^{\circ}$ EF e do $1^{\circ}$ EM de 2008 e 2009

Gráfico 1 - Taxa de não aprovação da escola A1 de 2003 a 2005 .

Gráfico 2 - Taxa de não aprovação da escola A2 de 2003 a 2005 .

Gráfico 3 - Taxa de não aprovação da escola A3 de 2003 a 2005 .

Gráfico 4 - Taxa de não aprovação da escola A4 de 2003 a 2005 .

Gráfico 5 - Taxa de não aprovação da escola A5 de 2003 a 2005 .

Gráfico 6 - Taxa de não aprovação da escola A6 de 2003 a 2005 .

Gráfico 7 - Taxa de não aprovação da escola B1 de 2003 a 2005 .

Gráfico 8 - Taxa de não aprovação da escola B2 de 2003 a 2005 .

Gráfico 9 - Taxa de não aprovação da escola B3 de 2003 a 2005 .

Gráfico 10 - Taxa de não aprovação da escola C1 de 2003 a 2005

Gráfico 11 - Taxa de não aprovação da escola D1 de 2003 a 2005

Gráfico 12 - Taxa de não aprovação da escola E1 de 2003 a 2005

Gráfico 13 - Taxa de não aprovação da escola F1 de 2003 a 2005

60

Gráfico 14 - Taxa de não aprovação da escola G1 de 2003 a 2005 
Gráfico 16 - Número de alunos por série dos quatro ciclos de série da escola A1 f........................................................... 66

Gráfico 17 - Número de alunos por série dos quatro ciclos de série da escola $\mathrm{A} 2$

Gráfico 18 - Consolidado dos quatro ciclos da Escola A1 com a porcentagem na composição das séries

Gráfico 19 - Consolidado dos quatro ciclos da Escola A2 com a porcentagem na composição das séries

Gráfico 20 - Consolidado dos quatro ciclos da Escola A1 e A2 com a proporção e o número absoluto de alunos: concluintes, reprovados, reprovados e transferidos no mesmo ano e dos transferidos

Gráfico 21 - Médias das reprovações e transferências nos quatro ciclos de série da escola A1

Gráfico 22 - Médias das reprovações e transferências nos quatro ciclos de série da escola A2

Gráfico 23 - Consolidado dos quatro ciclos da Escola A1 e A2 com os alunos que foram reprovados e seus destinos 